Scientific Paper

\title{
Study the Anti-MUC1 antibody-based iron oxide nanoparticles on three- dimension spheroid and breast cancer (MCF-7) cell imaging
}

\author{
Pegah Moradi KHANIABADI ${ }^{1}$, Daryoush ShaHBAZI-GaHROUEI ${ }^{2, a}$, Amin Malik SHAH ABDUL MAJID ${ }^{3}$, Bita MoRADI \\ KHANIABADI $^{4}$ \\ ${ }^{1}$ School of Physics, Universiti Sains Malaysia11800, Pinang, Malaysia \\ ${ }^{2}$ Dept. of Medical Physics, School of Medicine, Isfahan University of Medical Sciences, Isfahan, Iran \\ ${ }^{3}$ School of Pharmaceutical Sciences, Universiti Sains Malaysia, 11800 Penang, Malaysia \\ ${ }^{4}$ Child Growth and Development Research Center, Research Institute for Primordial Prevention of Non-communicable \\ Disease, Isfahan University of Medical Sciences, Isfahan, Iran \\ ${ }^{a}$ E-mail address: shahbazi@med.mui.ac.ir
}

(received 28 December 2018; revised 12 March 2019; accepted 26 March 2019)

\begin{abstract}
Non-invasive methods for breast cancer detection in early stages may help to increase the survival rate of patients. This study aimed to evaluate the application of Anti-MUC1 antibody-based iron oxide nanoparticle (SPIONs-C595) which was assessed in vivo as a molecular imaging probe for breast cancer (MCF-7) detection using MRI. Nine groups of female NRC NU/Nu mice (each group of 3), 6 to 8 weeks old were used and MCF-7 cells were injected subcutaneously into both flanks of nude mice. After two weeks the mice received an intravenous injection of different concentrations of SPIONs-C595. The uptake ability of SPIONs-C595 on three-dimension (3D) macrostructure is exploited a modified hanging drop method using Prussian blue for MCF-7 cells. The iron content was measured in liver, kidney, spleen, and tumor. The MR imaging features and biodistribution of nanoprobe was also investigated. The MR images obtained from digested tumor after nanoprobe administration in different time-period revealed that enhancement of $T_{1}$ and $T_{2}$ relaxation time. Moreover, the storage stability test was shown great application and no sedimentation of nanoparticles within two months storage at $4^{\circ} \mathrm{C}$. Additionally, great validation of SPIONs-C595 on the 3D spheroid of MCF-7 was observed. The biodistribution analysis showed that iron content of the spleen was more than the other studied organs. These results highlighted the feasibility of an in-vivo model for detection of breast cancer MUC1 expression. Current researches are ongoing to further enhancement of relaxation times for classification of MUC1 status using clinical specimens.
\end{abstract}

Key words: SPIONs; MR imaging; MCF-7 cells; C595 monoclonal antibody.

\section{Introduction}

Magnetic resonance imaging has been useful to detect and distinguish various types of cancers [1-3]. Molecular imaging has recently emerged enabling the discovery and identification of new molecular pathways of living organisms in a noninvasive fashion and is useful for early detection of cancer [4, 5]. In this regard, the accuracy and reliability of MR imaging, using contrast agents is significantly better in women with high risk for breast cancer, because the contrast of the specific region in the image will be enhanced due to the effect of the contrast agent $[5,6]$.

MR imaging contrast agents are used primarily to increase the sensitivity of the MR imaging to detect and characterise tumor at early stages [7-9]. In recent years, antibodies are widely used in cancer diagnosis in vitro and in vivo [10-14]. One of the targets of breast cancer cells is the breast-specific membrane antigen (MUC1). MUC1 is an over-expressed on the majority of human epithelial cells of adenocarcinomas resulting in the exposure of new peptide epitopes in addition to oligosaccharides, which are used as novel target molecules. MUC1s exist in the ductal epithelial cells which plays a major role in the protection and lubrication of normal tissue that has identical amino acid sequences. Therefore, the MUC1 antigen may be a useful diagnostic target to minimize the growth of incurable cancers $[15,16]$.

In the past decades, significant approaches have been made in the development and application of MR imaging and its role in early stage detection of breast cancer $[6,13]$. Moreover, the presence of any MUC1 in most of the tumor cells is associated with an improved prognosis. Shahbazi-Gahrouei and Abdolahi have been studied on developing ovarian cancer (OVCAR3) by C595 monoclonal antibody which was coupled with SPIONs $[8,11]$. They reported that the SPIONs-C595 mAb could attach

(C) 2019 Pegah Moradi Khaniabadi, Daryoush Shahbazi-Gahrouei, Amin Malik Shah Abdul Majid, Bita Moradi Khaniabadi. This is an open access article licensed under the Creative Commons Attribution-NonCommercial-NoDerivs License (http://creativecommons.org/licenses/by-nc-nd/3.0/). 
to MUC1 which could detect ovarian cancer cells using MR imaging.

C595 mAb contains immunogenicity due to its murine origin in addition to poor tumor penetration characteristics due to its large molecular size (150 kDa). Superparamagnetic iron oxide nanoparticles (SPIONs) provide a strong contrast effect in $\mathrm{T}_{1}$ and $\mathrm{T}_{2}$-weighted $\mathrm{MR}$ images due to its different contrasting mechanisms [17-19]. However, as a result of the tremendous progress in nanotechnology, many scientists have recently developed new nanoparticulate MR imaging contrast agents that have further improved image contrast with additional functions [20-22]. Hence, the production and evaluation of magnetic nanoprobe (SPIONs-C595) and its application as an MR imaging contrast agent for targeted molecular imaging of breast cancer cells was investigated. Although many in vivo studies have been done, however, the investigation of tumour spheroid has attracted the attention of researchers to study the 3D tumor model. It was revealed that the size of spheroids is 200-500 $\mathrm{mm}$ in diameter [23,24].

In many studies, Shahbazi-Gahrouei and co-workers fabricated and designed the mentioned nanoprobe and investigated its characterization, its $T_{1}$ and $T_{2}$ relaxation times determination and its cytotoxicity effects under in vitro conditions in breast cancer (MCF-7) cells [25-27].

The main scope of this study is to investigate SPIONs-C595 as an MR imaging contrast agent for targeted molecular imaging of MUC1-expressing breast cancer cells (MCF-7) under in vivo conditions. Consequently, it is tested with a human breast (MCF-7) xenograft to investigate its pharmacokinetics, biological distribution, $\mathrm{T}_{1}$ and $\mathrm{T}_{2}$ relaxation time measurements and signal enhancement at optimal doses in cancerous nude mice at post-injection.

\section{Materials and methods}

\section{Chemicals}

All chemicals were purchased from Sigma-Aldrich. NanomagD-spio $20 \mathrm{~nm}$ nanoparticles (surface $\mathrm{COOH}$ ) were purchased from Micromode Company (micromod Partikeltechnologie $\mathrm{GmbH}$, Rostock, Germany). MiniMACS separator in addition to $\mathrm{C} 595$ mab was purchased from Miltenyi Biotech $\mathrm{GmbH}$, Germany.

\section{MCF-7 cells}

MCF-7 cell was obtained from ATCC, USA and were routinely cultured in pre-warmed DMEM $\left(37^{\circ} \mathrm{C}\right.$ in water bath) supplemented with $10 \%$ of fetal bovine serum (FBS), antibiotics (100 IU/ml penicillin and $100 \mu \mathrm{g} / \mathrm{ml}$ streptomycin), $1 \% \mathrm{v} / \mathrm{v}$ essential amino acids and $2 \mathrm{mM}$ L-glutamine. The cells were grown in culture flasks with media $(15 \mathrm{ml}$ media/175 $\mathrm{cm}^{3}$ ) and incubated at $37^{\circ} \mathrm{C}$, in a humidified atmosphere of $95 \% / 5 \%$ air $/ \mathrm{CO}_{2}$. Cells were harvested after reaching approximately $80 \%$ confluence where the cells were presented as a monolayer.

\section{Animals}

The animal studies were performed, using 27 female NRC NU/NU mice, [5] 6 to 8 weeks old with the mean weights of $23.67 \pm 1.40$ g. Animals were randomly divided into nine groups of three. Each group was housed in a cage in humidity and temperature controlled isolated animal house at the EMAN laboratory, School of Pharmaceutical Sciences, USM. The sterilized standard mouse chow and water was provided ad libitum to all mice. The experimental procedure and the use of animals were approved by the Animal Ethics Committee (AECUSM) before the commencement of experiments [2014/ (94) (653)].

\section{Nanoprobe preparation}

Shahbazi-Gahrouei and co-workers fabricated and designed the mentioned nanoprobe and investigated its characterization, its $\mathrm{T}_{1}$ and $\mathrm{T}_{2}$ relaxation times determination and its cytotoxicity effects under in vitro conditions in breast cancer (MCF-7) cells. Characterization of SPIONs-C595 nanoprobe was confirmed using of FT-IR, XRD, TEM, SEM-EDAX and Zetasizer [25, 26]. An MTT assay conducted to confirm that there was no cytotoxicity effect of nanoprobe. Prussian blue staining confirmed that significant contrast enhancement of breast cancer could still be clearly seen even $24 \mathrm{hr}$ post-injection, due to the retention of SPIONs-C595 in the cytoplasm of cancer cells. In vitro results showed good binding of $200 \mu \mathrm{g} \mathrm{Fe} / \mathrm{ml}$ nanoprobe towards MCF-7 breast cancer cells [27].

The schematic diagram of SPIONs-C595 preparation is explained in Figure 1a. In brief, A $500 \mu$ of the nanomag-Dspio was mixed with a working solution, $0.6 \mathrm{mg}(3 \mu \mathrm{mol}) \mathrm{N}$ ethyl-N-(3 dimethyl aminopropyl) carbodiimide hydrochloride (EDC) and $1.2 \mathrm{mg}(10 \mu \mathrm{mol}) \mathrm{N}$ - hydroxyl succinimide (NHS) in $125 \mu \mathrm{l} 0.5 \mathrm{M}$ 2-(N-morpholino) ethanesulfonic acid (MES) buffer. The suspension was mixed for $90 \mathrm{~min}$ at room temperature by a shaker. Then, the suspension was washed twice with $1 \mathrm{ml} \mathrm{PBS}, \mathrm{pH}=7.4$ through $\mathrm{MS}$ column. To activate the columns before adding the mixture, the columns were washed three times with PBS, $\mathrm{pH}=7.4$. A $100 \mu \mathrm{MUC1}$ (C595) mab was added for nanoparticles activation and the suspension was shacked for $3 \mathrm{~h}$. Finally, the $30 \mu \mathrm{l}$ glycine was added for $30 \mathrm{~min}$ to quench the reaction of the final mixtures and the suspension was washed two times with $1 \mathrm{ml}$ PBS through MS column. By passing the external magnetic field, conjugated nanoparticles were retained in the magnetic field of the separation column. Therefore, non-conjugated mab was washed out through the column as a negative fraction. Finally, by removing the column from the magnetic field, the suspension was eluted as the enriched positive fraction. Of course, when the carboxyl groups of the SPIONs are activated the C595 may become attached to the SPIONs surface. Moreover, the storage stability test was also conducted to show the efficacy of the nanoprobe after long-time of storage period before in vivo study. 


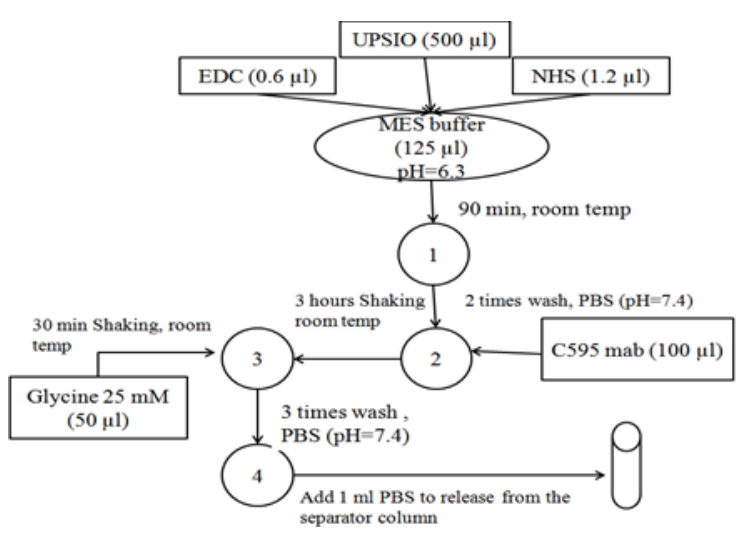

(a)

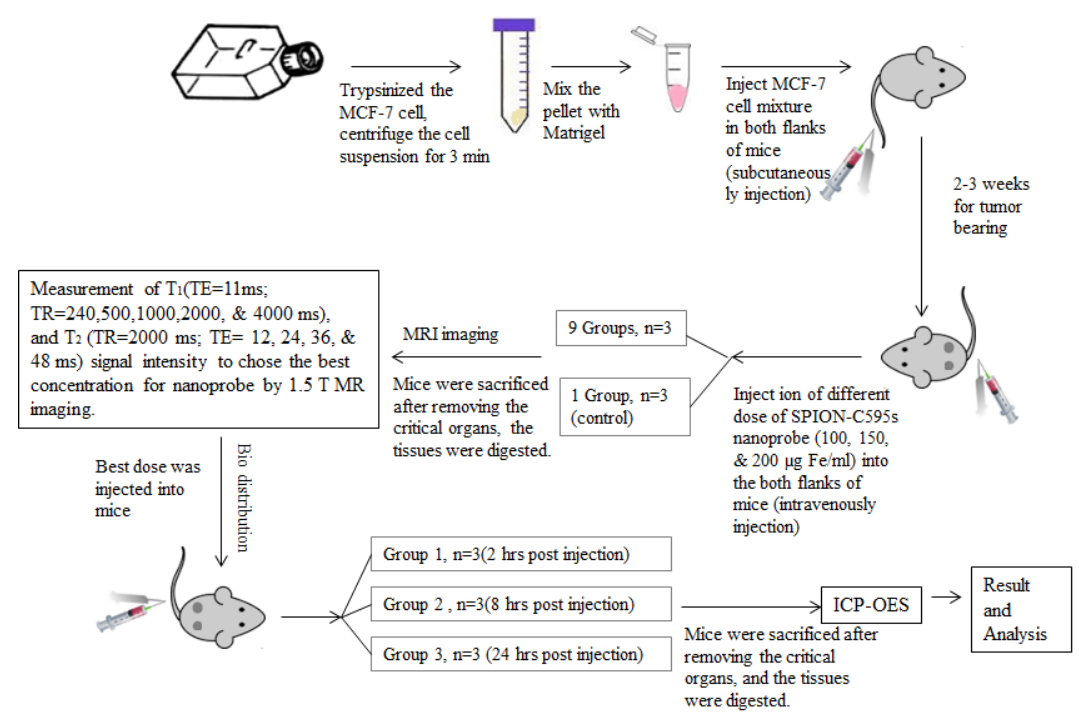

(b)

Figure 1. (a) Schematic diagram of SPIONs-C595 preparation and (b) in vivo study.

\section{Prussian blue histology staining for assessing of MCF-7 spheroid}

The novel development of iron staining method on MCF-7 3D spheroid-based was done using the modification of the method of Jafari and colleges and the method which they conducted on 2D cell monolayer of MCF-7 [27]. Briefly, MCF-7 cells $\left(2.5 \times 10^{5} \mathrm{cells} / \mathrm{ml}\right)$ were cultured in DMEM. The confluent cultures were trypsinized, subsequently washed two times in phosphate buffered saline and resuspended in the $2 \mathrm{ml}$ medium. The cells were centrifuged for 3 minutes and $1000 \mathrm{rpm}$. The media was removed and $1 \mathrm{ml}$ fresh DMEM, which contained $20 \%$ FBS and $0.25 \%$ methylcellulose was added. The pallet was mixed well with the medium to get the homogenous cell suspension. Drops (20 $\mu \mathrm{l})$ of medium containing MCF-7 (5000 cells/ drop) were put on the cover of 96-well plate, which were inverted over a plate containing $10 \mathrm{ml}$ DMEM medium in total to maintain humidity [28]. Then, 96-well plate was incubated overnight for sedimentation. After observing cellular aggregates, the medium was removed carefully, and $20 \mu \mathrm{l}$ of two different concentrations of the SPION-C595 (100 and 200 $\mu \mathrm{g} \mathrm{Fe} / \mathrm{mL}$ ) added to the MCF-7 spheroids. Then, the cells were incubated for 6 hours with the compounds. After the incubation time, the medium was removed carefully, and the spheroids were washed 2 times with $20 \mu \mathrm{l}$ of PBS. Then, $20 \mu \mathrm{l}$ of $4 \%$ PFAwas added and the spheroid was incubated overnight for fixation. Again, the fixed spheroids were washed three times with $20 \mu \mathrm{l}$ PBS, and $20 \mu \mathrm{l}$ of working solution was added for $30 \mathrm{~min}$ at room temperature $\left(25^{\circ} \mathrm{C}\right)$ in a darkroom. After the incubation, the spheroids were washed two times with PBS, and were examined under the inverted microscope.

\section{Storage stability test}

To determine the colloidal stability and the stability of conjugated SPION, The nanoprobe (SPION-C595) was stored at 2 to $8^{\circ} \mathrm{C}$. After defined time points ( 1 day, 1 week, 2 weeks, 1 month and 2 months) the iron staining was carried out at 2Dmonolayer cell culture MCF-7 [29,30].

\section{MR signal intensity enhancement}

MR signal intensity enhancement of prepared samples was performed by Signa HDxt 1.5 T (GE Healthcare, Wisconsin, USA). The image of the samples was obtained using spin-echo sequence. The subcutaneous injection method was utilized to inject the breast cancer cells, MCF- $7(2.5 \times 106,300 \mu 1$ in $1: 1$ matrigel) into both flanks of nude mice. Two to three weeks after tumor implantation, when the tumor diameter was 3 to 5 $\mathrm{mm}$ (mean weight of tumor was $50 \mathrm{mg}$ ), the mice received intraperitoneally (i.p) injection of different concentrations of SPIONs-C595. Figure 2 showed the size and location of tumors in NRC NU/NU mice. Schematic diagram of in vivo study is shown in Figure 1b.

All concentrations were diluted in physiological saline to a final concentration as injected in bolus doses 100, 150 and 200 $\mu \mathrm{g} \mathrm{Fe} / \mathrm{g}$ organ. The last group was a control group. The total injected volume was $1.08 \mathrm{ml}$. The animals were sacrificed by an overdose of pentobarbital sodium 2, 8, $24 \mathrm{~h}$ post i.p injection, followed by the removal of the critical organs (tumor, kidney, liver, spleen).

MR image of the tumor was acquired before the administration of different concentrations of SPIONs-C595, as well as post i.p infusion. A $\mathrm{T}_{1}$-weighted imaging method was conducted to obtain all of the images using multi-spin-echo pulse sequence technique, with TE value of $11 \mathrm{~ms}$, TR values $240,500,1000,2000,4000 \mathrm{~ms}$. To evaluate the $\mathrm{T}_{2}$-weighted 


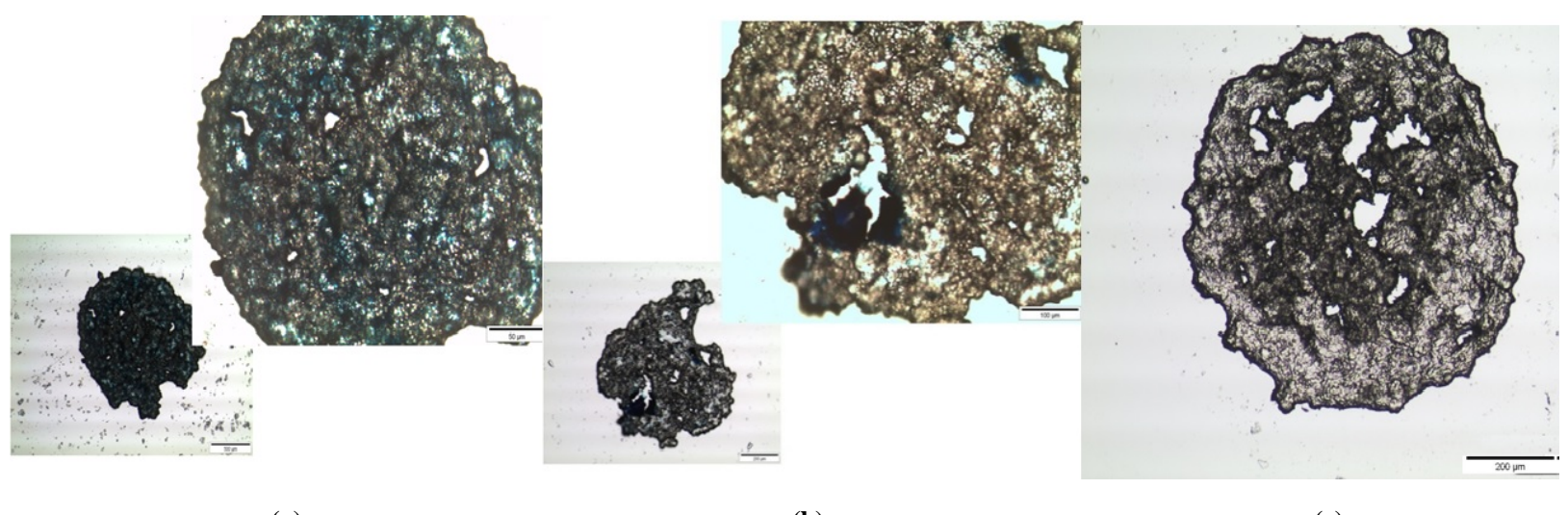

(a)

(b)

(c)

Figure 2. 3D cultures of MCF-7 spheroids. (a) and (b), are the images of MCF-7 spheroid after incubation with 200 and $100 \mu \mathrm{g}$ Fe/ml of the compound after $6 \mathrm{~h}$ at 4 and $40 \times$ magnifications. (c) is the control at $20 \times$ magnifications.

images, the values of echo times ( $\mathrm{TE}=12,24,36,48 \mathrm{~ms})$ and repetition time ( $\mathrm{TR}=2000 \mathrm{~ms}$ ) were used. All digested tumors were imaged by a $1.5 \mathrm{~T}$ MRI system. The samples were put in the Eppendorf tube box holder and to avoid the noise the box was filled with $1 \%$ agarose gel. Finally, samples were placed at the centre of the standard circular polarized head coil. The field of view of $20 \mathrm{~mm}$ and matrix size of $256 \times 256$ was applied. The slice thickness or number of cuts was $3 \mathrm{~mm}$. To determine the slope of the regression line, the relaxation rate was calculated by plotting $1 / T_{1}$ and $1 / T_{2}$ values versus different concentrations of nanoparticle and nanoprobe.

\section{Iron concentration measurements}

The SPIONs-C595 content was measured by inductively coupled plasma optical emission spectroscopy (ICP-OES) in sample solutions of harvested tissues which they prepared by acid digestion of Tamat et al. which described previously [6]. Each ICP-OES experiment was performed at least three times after acid digestion procedure. The percentage of iron concentration (mg/g organ) was obtained as biodistribution of the conjugate in the studied organs.

\section{Biodistribution measurement}

The SPION-C595 content in organs was measured on the solution obtained from acid digestion of the required tissue by ICP-OES. Briefly, a weighed sample of tissue $(50-100 \mathrm{mg})$ in a polyethylene vial, $0.3 \mathrm{ml}$ of $72 \%$ perchloric acid was carefully added and the contents swirled to mix. $0.6 \mathrm{ml}$ of $32 \%$ hydrogen peroxide was added and the clean glass vials placed in the shaking bath overnight at $25^{\circ} \mathrm{C}$, the glass vial contents were clear and colourless. The samples were diluted to $3 \mathrm{ml}$ of distilled water and filtered through a $0.45 \mu \mathrm{m}$ Millipore filter before being introduced into the ICP-OES. The $238.204 \mathrm{~nm}$ atomic emission line of iron was chosen for the ICP-OES analysis [6]. The percentage of iron concentration (mg per gram of organ) was obtained as biodistribution of the conjugate in the studied organs.

\section{Statistical analysis}

Data were analysed using IBM SPSS (IBM Crop. 2011. IBM statistics for windows, version 20.0. NY, EUA). MannWhitney tests were performed to compare means between two independent groups which did not follow normal distribution. Kruskal-Wallis tests were performed to determine the mean differences between more than two independent groups with non-normal distribution. P-values $<0.05$ were significant.

\section{Results}

\section{General Aspects}

All animals tolerated the procedures well, including tumor growth and response to nanoprobe. No adverse effects were observed after i.p injection of nanoprobe and no animal death was recorded during tumor growth or post-injection.

\section{Prussian blue histology stanning for assessing of MCF 7 spheroid}

The hanging drop spheroid assay is a useful assay that can be utilized to investigate cell-cell cohesion and cell-substratum adhesion throughout the generation of 3D spheroids in physiological situations. The spheroid aggregate mimics the biophysical aspect of a solid tumor and can provide useful information with respect to the ability of a particular drug molecule or drug formulation to penetrate into the solid tumor. To overcome the loss of tissue-specific properties is common for cells grown in two-dimensional monolayer cultures. Therefore, this assay is required to assess the effect a particular drug molecule or drug formulation on tissue in its natural, three-dimensional structure. In the present study, iron staining was applied to the MCF-7 spheroid to confirm the presence of the contrast agent on the solid tumor. Figure 2 shows the iron staining on the spheroids of MCF-7 cellular aggregates that had developed in vitro in the hanging drop assay. After complete sedimentation, the cellular aggregates were checked under the microscope. At a high SPIONs-C595 concentration (200 $\mu \mathrm{g}$ $\mathrm{Fe} / \mathrm{ml}$ ), a high amount of attachment was observed on the 
MCF-7 spheroid, while at lower concentrations, (50 and $25 \mu \mathrm{g}$ $\mathrm{Fe} / \mathrm{ml}$ ), a lower amount of attachment was observed. The results show that with the nanoprobe at a concentration of 200 $\mu \mathrm{g} \mathrm{Fe} / \mathrm{ml}$, there was a greater labelling on SPIONs-C595 spheroids compared to the control spheroid.

\section{Storage stability test}

Figure 3 implies that the SPIONs-C595 has good dispersity and stability under physiological conditions. The nanoprobe reaming suspended in solution form at desired storage temperature up to two months. The biocompatibility of SPIONs-C595 on stained MCF-7 showed that the nanoprobe was abled to attach onto the targeted MUC1. The SPIONsC595 nanoprobe could be a great possible candidate as an MR contrast agent for in vivo biomedical applications.

\section{MR imaging}

Figure 4 showed $T_{1}$ - and $T_{2}$-weighted images of digested breast tumors after administration of different doses of SPIONs-C595. Table 1 and 2 represents the change of $T_{1}$ and $\mathrm{T}_{2}$ values after the administration of SPIONs-C595 at 100, 150 and $200 \mu \mathrm{g} \mathrm{Fe} / \mathrm{g}$ organ, compared with the control group. For each concentration, three post-injection times were considered (2, 8, and $24 \mathrm{~h})$.

\section{Relaxation rates}

According to Table 3, the highest relaxation rate ratio (8.03) was obtained from the SPIONs-C595 at a concentration of 200 $\mu \mathrm{gFe} / \mathrm{ml}$ after $2 \mathrm{~h}$ post-injection. This value was higher than other post-injection times at different concentrations as well as the control (2.57).

As can be seen from Table 3, the relaxation rate ratio of SPIONs-C595 at all concentrations after all post-injection times were higher than the control. High relaxation rate ratios were obtained at a concentration of $150 \mu \mathrm{mol} / \mathrm{g}$ organ after 2 and $8 \mathrm{~h}$ post-injection.
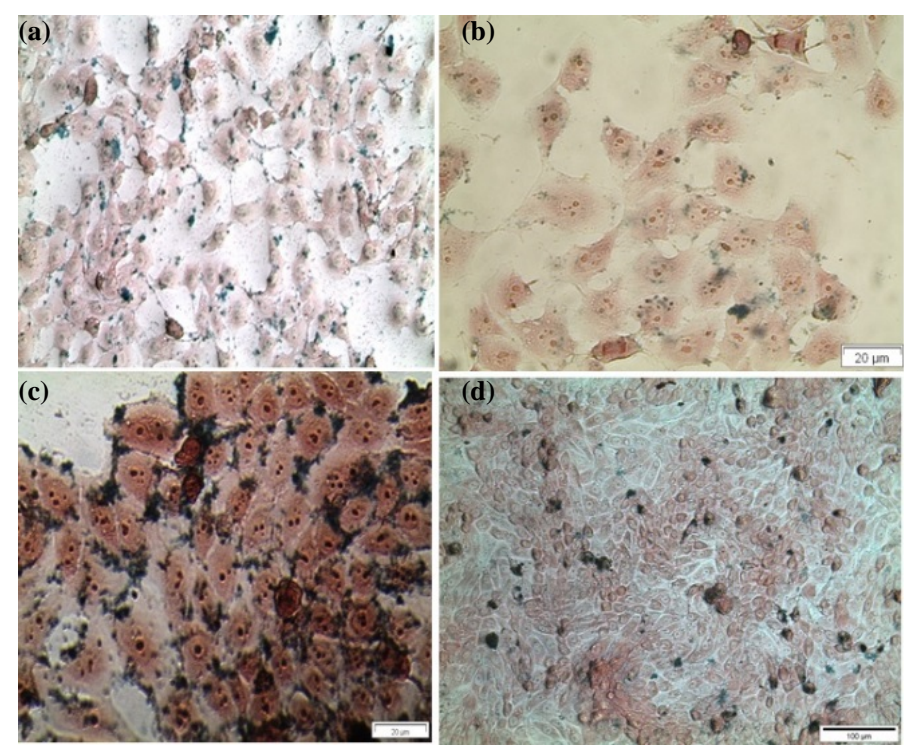

Figure 3. MCF-7 cells exposed to $200 \mu \mathrm{g}$ Fe/ml SPIONs-C595 for 6 $h$ after several periods of storage. (a), (b), (c), and (d) are the images of MCF-7 cells after incubation with one day, two weeks, one month and two months, respectively. Iron content was determined by the histological Prussian blue reaction. Images are at 10x magnification.

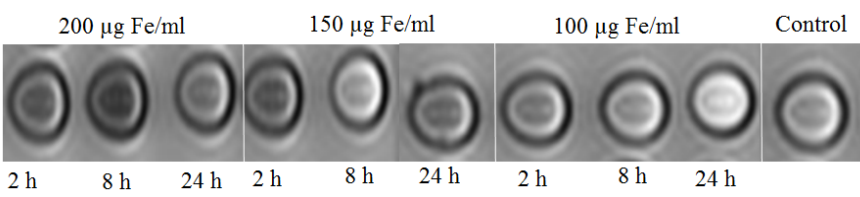

(a)

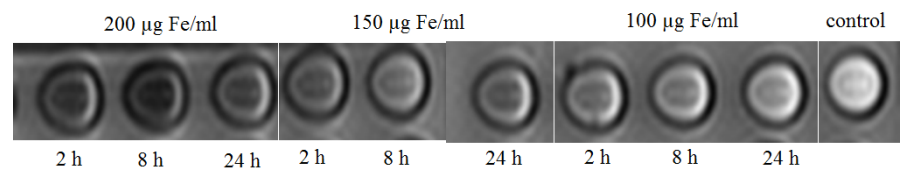

(b)

Figure 4. $T_{1}$-weighted (a) and $T_{2}$-weighted (b) images of samples which prepared by acid digestion method after administration of different doses of 100, 150 and $200 \mu \mathrm{gFe} / \mathrm{ml}$ of SPIONs-C595 and 2, 8 and 24 h post injection SPIONs-C595.

Table 1. Spin-lattice $\left(\mathrm{T}_{1}\right)$ relaxation times (Mean $\left.\pm \mathrm{SD}\right)(\mathrm{ms})$ of different doses of SPIONs-C595 in breast tumors after different postinjection times.

\begin{tabular}{|c|c|c|c|c|}
\hline $\begin{array}{c}\text { SPION-C959 dosage } \\
(\mu \mathrm{gFe} / \mathrm{g} \text { organ })\end{array}$ & Administration time (hour) & $\operatorname{Mean} \pm \mathrm{SD}^{\mathrm{a}}$ & P-value ${ }^{b}$ & P-value ${ }^{c}$ \\
\hline \multirow{3}{*}{ 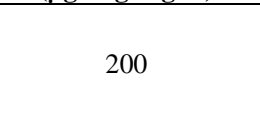 } & 2 & $1638.89 \pm 375.77$ & 0.653 & \multirow{3}{*}{0.109} \\
\hline & 8 & $956.89 \pm 153.47$ & $0.046^{*}$ & \\
\hline & 24 & $1555.55 \pm 509.17$ & 0.653 & \\
\hline \multirow{3}{*}{150} & 2 & $1202.11 \pm 142.77$ & $0.046^{*}$ & \multirow{3}{*}{0.188} \\
\hline & 8 & $1638.89 \pm 375.77$ & 0.653 & \\
\hline & 24 & $1805.55 \pm 636.47$ & 0.653 & \\
\hline \multirow{3}{*}{100} & 2 & $1163.23 \pm 75.65$ & $0.046^{*}$ & \multirow{3}{*}{0.353} \\
\hline & 8 & $1559.52 \pm 391.77$ & 0.817 & \\
\hline & 24 & $2000.00 \pm 1201.85$ & 0.653 & \\
\hline \multicolumn{2}{|c|}{ Control } & $1507.93 \pm 37.46$ & - & - \\
\hline
\end{tabular}

${ }^{\mathrm{a}}$ Mean (standard deviation) of $\mathrm{T}_{1}(\mathrm{~ms})$ values.

${ }^{\mathrm{b}}$ Using Mann-Whitney $\mathrm{U}$ test, $\mathrm{T}_{1}(\mathrm{~ms})$ values of administration time versus control group.

${ }^{\mathrm{c}}$ Using Kruskal-Wallis test, $\mathrm{T}_{1}(\mathrm{~ms})$ values of SPION-C595( $\mu \mathrm{gFe} / \mathrm{g}$ organ) among administration times $(2,8,24 \mathrm{~h})$.

*Statistically significant at level of $5 \%$. 
Table 2. Spin-spin $\left(\mathrm{T}_{2}\right)$ relaxation times (Mean $\left.\pm \mathrm{SD}\right)(\mathrm{ms})$ of different doses of SPIONs-C595 in the breast tumors after different postinjection times.

\begin{tabular}{|c|c|c|c|c|}
\hline $\begin{array}{c}\text { SPION-C595 dosage } \\
(\mu \mathrm{g} \mathrm{Fe} / \mathrm{g} \text { organ })\end{array}$ & Incubation time (hour) & Mean \pm SD $^{a}$ & P-value ${ }^{\text {b }}$ & $P$-value ${ }^{c}$ \\
\hline \multirow{3}{*}{ 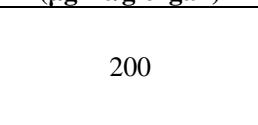 } & 2 & $203.53 \pm 62.93$ & $0.049^{*}$ & \multirow{3}{*}{0.430} \\
\hline & 8 & $279.21 \pm 61.56$ & $0.049^{*}$ & \\
\hline & 24 & $318.63 \pm 50.12$ & $0.049^{*}$ & \\
\hline \multirow{3}{*}{150} & 2 & $241.42 \pm 168.27$ & $0.049^{*}$ & \multirow{3}{*}{0.113} \\
\hline & 8 & $293.59 \pm 171.16$ & $0.049^{*}$ & \\
\hline & 24 & $578.41 \pm 193.15$ & 0.827 & \\
\hline \multirow{3}{*}{100} & 2 & $299.82 \pm 99.28$ & $0.046^{*}$ & \multirow{3}{*}{0.065} \\
\hline & 8 & $338.10 \pm 36.10$ & $0.049^{*}$ & \\
\hline & 24 & $586.39 \pm 307.08$ & 0.513 & \\
\hline \multicolumn{2}{|c|}{ Control } & $569.67 \pm 175.42$ & - & - \\
\hline
\end{tabular}

${ }^{\mathrm{a}}$ Mean (standard deviation) of $\mathrm{T}_{2}(\mathrm{~ms})$ values.

${ }^{\mathrm{b}}$ Using Mann-Whitney $\mathrm{U}$ test , $\mathrm{T}_{2}(\mathrm{~ms})$ values of administration time versus control group.

${ }^{\mathrm{c}}$ Using Kruskal-Wallis test, $\mathrm{T}_{2}(\mathrm{~ms})$ values of SPION-C59( $\mu \mathrm{g}$ Fe/g organ) among administration times $(2,8,24 \mathrm{~h})$.

* Statistically significant at level of $5 \%$.
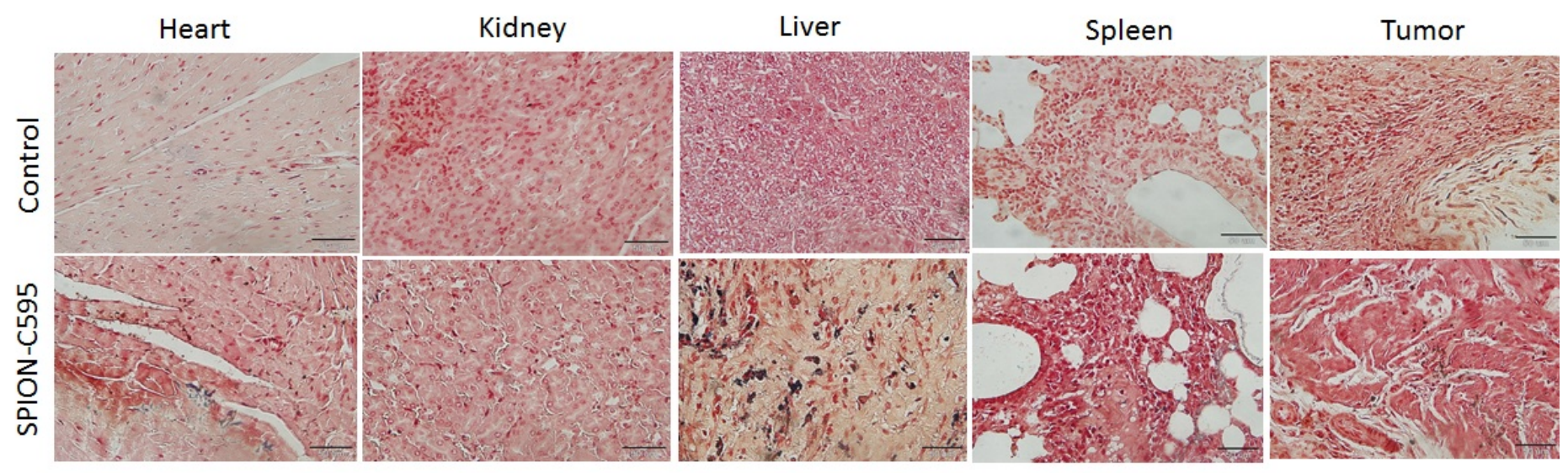

Figure 5. Prussian blue staining of the different tissues before and after administration of SPIONs-C595 at $600 \mu \mathrm{g} \mathrm{Fe} / \mathrm{ml}$. The blue colours show the iron content.

Table 3. The $R_{1}, R_{2}$ and relaxation rate ratio of different doses of SPIONs-C595 in breast tumor after different post-injection times.

\begin{tabular}{cccc}
\hline \hline $\begin{array}{c}\text { Dose ( } \mathbf{\mu m o l} / \mathbf{g b w} \text { mouse)/ } \\
\text { Post-injection time (hour) }\end{array}$ & $\mathbf{1 / T 2}\left(\mathbf{s}^{-\mathbf{1}}\right)$ & $\mathbf{1 / T 1}\left(\mathbf{s}^{-\mathbf{1}}\right)$ & $\begin{array}{c}\text { Relaxation rate } \\
\text { ratio }\end{array}$ \\
\hline 200,2 & 0.0049 & 0.00061 & 8.03 \\
200,8 & 0.0035 & 0.00100 & 3.50 \\
200,24 & 0.0031 & 0.00064 & 4.84 \\
150,2 & 0.0041 & 0.00083 & 4.93 \\
150,8 & 0.0034 & 0.00061 & 5.57 \\
150,24 & 0.0017 & 0.00055 & 3.09 \\
100,2 & 0.0033 & 0.00085 & 3.88 \\
100,8 & 0.0029 & 0.00064 & 4.53 \\
100,24 & 0.0017 & 0.00050 & 3.40 \\
Control & 0.0017 & 0.00066 & 2.57 \\
\hline \hline
\end{tabular}

Figure 5 and Table 4, showed SPIONs-C595 bindings to the breast (MCF-7) cancer cells and other harvested organs. The iron contents of the breast cancer cells at 2 and $24 \mathrm{hrs}$ postinjection were almost the same, but at $8 \mathrm{hr}$ post-injection, the iron content slightly dropped due to the size of the tumors. Fewer nanoprobes could be detected on smaller tumors.
Table 4. Iron content of extracted organs $(n=3)($ Mean \pm SD) after injected 200 ( $\mu \mathrm{gFe} / \mathrm{g}$ organ) doses of SPIONs-C595 at different post-injection times.

\begin{tabular}{cccc}
\hline \hline Organ & $\begin{array}{c}\text { Administration time } \\
\text { (hour) }\end{array}$ & Mean \pm SD & P-value $^{\mathbf{b}}$ \\
\hline \multirow{3}{*}{ Tumour } & 2 & $0.14 \pm 0.07$ & \\
& 8 & $0.11 \pm 0.01$ & 0.066 \\
& 24 & $0.28 \pm 0.01$ & \\
Spleen & 2 & $2.77 \pm 0.35$ & 0.118 \\
& 8 & $2.61 \pm 0.73$ & \\
\hline \multirow{3}{*}{ Liver } & 24 & $1.59 \pm 0.39$ & $0.027^{*}$ \\
& 2 & $0.71 \pm 0.06$ & \\
\hline \multirow{3}{*}{ Kidney } & 8 & $0.83 \pm 0.05$ & $0.027^{*}$ \\
& 24 & $1.00 \pm 0.03$ & \\
\hline \hline
\end{tabular}

$\overline{{ }^{a}}$ Mean (standard deviation) of \% Iron content (ppm Fe/g organ).

${ }^{\mathrm{b}}$ Using Kruskal Wallis $\mathrm{H}$ test, \% Iron content (ppm Fe/g organ)among administration times $(2,8,24 \mathrm{~h})$.

*Statistically significant at level of $5 \%$. 


\section{Discussion}

The cellular uptake of SPIONs-C595 was assessed by ICPOES and Prussian blue staining, followed by the measurement of iron uptake by the cells. The results reported by Khaniabadi et al., showed that there was great cellular internalization of $200 \mu \mathrm{g} \mathrm{Fe} / \mathrm{ml}$ dose of nanoprobe on the MCF-7 cells [26,27].

To estimate the efficacy of any negative contrast agent, it is essential to calculate the $R_{2} / R_{1}$ ratio which is called the relaxation rate ratio. According to Table 1, the relaxation rate ratio of SPIONs-C595 at all concentrations after post-injection times was higher than the control. High relaxation rate ratios were obtained at a concentrations $150 \mu \mathrm{mol} / \mathrm{g}$ organ after 2 and $8 \mathrm{~h}$ post-injection, with the highest being obtained at a 200 $\mu \mathrm{mol} / \mathrm{g}$ organ after $2 \mathrm{~h}$ of post-i.p. injection of SPIONs-C595 into the mice. This result showed that the SPIONs-C595 had a greater influence on water protons as the concentration increased. Also, this phenomena is in agreement with the physics theory of contrast agents as the high relaxation rate of the contrast agent is critical for effective contrast-enhanced MR imaging [29]. For this reason, higher concentration was used for further procedure of the study.

The effect on magnetic relaxation can also be changed by increasing the particle size. Functionalize SPION particles can be linked to the MUC1 antigen of breast cancer cells, with their binding to the target causing the self-assembly of the particle and resulting in changes of relaxation times [30].

From MR images of prepared organs using acid digestion technique,[6] it can be seen that the $\mathrm{T}_{1}$ relaxation time was reduced at all doses, even at different post-injection times. For example, the $T_{1}$ relaxation times of treated tumors at nanoprobe doses of 100 and $150 \mu \mathrm{g} \mathrm{Fe} / g$ organ significantly decreased $23 \%$ and $20 \%$, respectively, a two hours post-injection $(\mathrm{P} \leq$ $0.05)$. In addition, a $37 \%$ reduction of $\mathrm{T}_{1}$ value was obtained in mice which were injected with $200 \mu \mathrm{g} \mathrm{Fe} / \mathrm{g}$ organ of nanoprobe, then sacrificed after $8 \mathrm{~h}(\mathrm{P} \leq 0.05)$.

The MR imaging of harvested organs results revealed that $2 \mathrm{~h}$ following the i.p injection of SPIONs-C595, a 47\%, 58\%, and $64 \%$ decrease of $\mathrm{T}_{2}$ relaxation times was observed for tumors injected with a SPIONs-C595 concentrations of 100 , $150,200 \mu \mathrm{gFe} / \mathrm{g}$ organs, respectively $(\mathrm{P} \leq 0.05)$ as indicated in Table 3. Moreover, $8 \mathrm{~h}$ of post-injection, the measurement of $\mathrm{T}_{2}$ relaxation showed that a $41 \%, 48 \%$, and $51 \%$ reduction of $\mathrm{T}_{2}$ values at doses of 100,150 , and 200 , respectively $(\mathrm{P} \leq$ 0.05). Finally, there were no significant reduction rates obtained for lower doses, however, $\mathrm{T}_{2}$ value of highest dose was decreased by $44 \%$ ( $\mathrm{P} \leq 0.05)$.

The signal intensity enhancement indicated significant binding of functionalized SPIONs on the breast cancer tumors compared to the untreated tumors. Statistically significant changes in the $T_{2}$ values were observed in the breast tumors ( $\leq$ $5 \mathrm{~mm}$ ) for all post-injection times of SPIONs-C595 at all concentrations $(\mathrm{P} \leq 0.05)$. The mean $\mathrm{T}_{2}$ value for the untreated nude mice was $596.67 \pm 175.42 \mathrm{~ms}$. Moreover, after the $24 \mathrm{~h}$ post-injection, there was no significant reduction of the $T_{2}$ value observed in the breast cancer cells with 100 and $150 \mu \mathrm{g}$ $\mathrm{Fe} / \mathrm{g}$ organ of SPIONs-C595 injected. Due to the fact that the number of targeted nanoparticle might be less at that given concentration, the probability of the accumulation of nanoprobe decreased due to the long circulation of the nanoparticles which caused their agglomeration (increasing the size) in the body. However, the significant decrease only occurred at post-injection of $200 \mu \mathrm{g} \mathrm{Fe} / \mathrm{ml}$ of SPIONs-C595 after post-injections (Figure 5) due to the existence of more targeted nanoparticles and less agglomeration possibility.

The results of iron content histology showed that by increasing the concentration of the compound, the possibility of attachment of the nanoprobe to the 3D spheroid MCF-7 (breast cancer) cells was increased compared to normal cells (Figure 2). As results demonstrated spleen has the highest amount and the breast tumor have the lowest amount. There was no significant change of iron content observed 2 to $24 \mathrm{~h}$ post-injection for tumor and spleen. This phenomenon might be due to the existence of unconjugated iron oxides, which easily agglomerate in bloodstreams. The agglomeration causes a decrease in the size of the nanoparticle, and nanoparticles larger than $100 \mathrm{~nm}$ could be caught by the macrophages in the spleen [31,32]. Thus, the iron content in the liver slightly increased as the post-injection time increased. After the spleen, the liver had the highest amount of iron which was significantly increased 2 to $24 \mathrm{~h}$ post-injections. The amount was about half of that of the spleen. Finally, the least amount of iron was detected in kidneys, with the iron content increased significantly 2 to $4 \mathrm{~h}$ post-injection. The iron content in the spleen showed the same results for all the groups, with the highest amount of detected iron compared to other tissues [10]. The final iron content was quite less compared to the iron content found in the spleens. A little amount of iron was detected in kidneys. In can be concluded that the excretion rout of suggested nanoprobe is through the kidneys as the iron contents in the liver, the iron content of the kidneys was slightly increased 2 to $24 \mathrm{~h}$ post-injection. Rodríguez E et al. [33] studied an iron-based $\mathrm{T}_{1}$ contrast agent made of ironphosphate complexes under in vitro and in vivo conditions. They reported that $T_{1}$-weighted images in mice have shown positive contrast enhancement of iron compounds which are in good agreement with the results of this study.

Today, the synthetic quantitative MR imaging through relaxometry ( $T_{1}$ and $T_{2}$ relaxation times measurements) modelling is desirable to distinguish more abnormalities, in particular using $\mathrm{T}_{1}$-weighted images [34]. In this study, using $\mathrm{T}_{1}$ and $\mathrm{T}_{2}$ relaxation times and signal intensity measurements revealed that MR imaging contrast enhancement of the SPIONs-C595 may be attributed to targeting breast cancer cells (MCF-7) which apparently increases the nanoprobe uptake by the tumor. In addition, the results of the stability test are confirmed that the nanoprobe is detecting significantly the cancer cells up to two months. Further work must be done to 
the determination of $T_{1}$ and $T_{2}$ values in the live animal and also it is recommended that the MR imaging be conducted with animal MR machines. This fact is the limitations of the present work which should be a highlight. Of course, to overcome this problem dissected tissue samples were sent for MR imaging. For in vivo MR imaging only the tumors were examined, but for biodistribution of nanoprobe measurements tumor, spleen, kidneys and liver were also analyzed.

\section{Conclusion}

It is concluded that with the satisfactory low levels of SPIONs in the liver, kidney, and spleen, as well as significant tumor uptake, SPIONs-C595 has shown considerable promise for further diagnostic applications in MR imaging. The observations of this study indicated that nanoparticles conjugated with C595 exhibit high dual ( $\mathrm{T}_{1}$ and $\left.\mathrm{T}_{2}\right)$ MR contrast potential and may be applied as a breast cancer detection agent at an early stage.

\section{Compliance with ethical standards}

In this study, the use of animals was approved by the Animal Ethics Committee (AECUSM) before the commencement of experiments [Reference number: 2014/ (94) (653)].

\section{Acknowledgments}

This study has been done as a joint $\mathrm{PhD}$ thesis project between Isfahan University of Medical Sciences and the Universiti Sains Malaysia. Authors would like to thanks all technicians for their support during this research.

\section{References}

[1] de Rooij M, Hamoen EH, Fütterer JJ, et al., Accuracy of multiparametric MRI for prostate cancer detection: a meta-analysis. American Journal of Roentgenology. 2014;202(2):343-351.

[2] Shahbazi-Gahrouei D. Novel MR imaging contrast agents for cancer detection. Journal of Research in Medical Sciences. 2009;14(3):141-147.

[3] Shahbazi-Gahrouei D, Rizvi S, Williams M, Allen BJ. In vitro studies of gadolinium-DTPA conjugated with monoclonal antibodies as cancer-specific magnetic resonance imaging contrast agents. Australasian Physics \& Engineering Sciences in Medicine. 2002;25(1):31-38.

[4] Padmanabhan P, Kumar A, Kumar S, et al. Nanoparticles in practice for molecular-imaging applications: An overview. Acta Biomaterialia. 2016;41:1-16.

[5] Shahbazi-Gahrouei D, Williams M, Rizvi S, Allen BJ. In vivo studies of Gd-DTPA-monoclonal antibody and gd-porphyrins: Potential magnetic resonance imaging contrast agents for melanoma. Journal of Magnetic Resonance Imaging. 2001;14(2):169-174.

[6] Abdolahi M, Shahbazi-Gahrouei D, Laurent S, et al. Synthesis and in vitro evaluation of MR molecular imaging probes using J591 mAb-conjugated SPIONs for specific detection of prostate cancer. Contrast Media and Molecular Imaging, 2013;8(2):175-184.

[7] Mirzaei M, Mohagheghi M, Shahbazi-Gahrouei D, Khatami A. Novel nanosized Gd3+-ALGD-G2-C595: in vivo dual selective MUC-1 positive tumor molecular MR imaging and therapeutic agent. J Nanomed Nanotechnol. 2012;3(7):147-152.

[8] Shahbazi-Gahrouei D, Williams M, Allen B. In vitro study of relationship between signal intensity and gadolinium-DTPA concentration at high magnetic field strength. Australasian Radiology. 2001;45(3):298-304.

[9] Shahbazi-Gahrouei D, Abdolahi M. A novel method for quantitative analysis of anti-MUC1 expressing ovarian cancer cell surface based on magnetic cell separation. Journal of Medical Sciences. 2012;12(8):256-266.

[10] Shahbazi-Gahrouei D, Abdolahi M. Superparamagnetic iron oxide-C595: Potential MR imaging contrast agents for ovarian cancer detection. Journal of Medical Physics. 2013;38(4):198-204.

[11] Shahbazi-Gahrouei D, Abdolahi M. Detection of MUC1-expressing ovarian cancer by C595 monoclonal antibody-conjugated SPIONs using MR imaging. The Scientific World Journal. 2013;2013:609151.

[12] Ghasemian Z, Shahbazi-Gahrouei D, Manouchehri S. Cobalt zinc ferrite nanoparticles as a potential magnetic resonance imaging agent: An in vitro study. Avicenna Journal of Medical Biotechnology. 2015;7(2):64-68.

[13] Zahraei M, Marciello M, Lazaro-Carrillo A, et al. Versatile theranostics agents designed by coating ferrite nanoparticles with biocompatible polymers. Nanotechnology. 2016;27(25):255702.

[14] Zahraei M, Monshi A, del Puerto Morales M, et al. Hydrothermal synthesis of fine stabilized superparamagnetic nanoparticles of $\mathrm{Zn}^{2+}$ substituted manganese ferrite. Journal of Magnetism and Magnetic Materials. 2015;393:429-436.

[15] Hattrup L, Gendler J. MUC1 alters oncogenic events and transcription in human breast cancer cells. Breast Cancer Research. 2006;8(4):R37.

[16] Wang L, Ma J, Liu F, et al. Expression of MUC1 in primary and metastatic human epithelial ovarian cancer and its therapeutic significance. Gynecologic Oncology. 2007;105(3):695-702. 
[17] Boult K, Borri M, Jury A, et al. Investigating intracranial tumour growth patterns with multiparametric MRI incorporating Gd-DTPA and USPIO-enhanced imaging. NMR in Biomedicine. 2016;29(11):1608-1617.

[18] Danhier P, Magat J, Levêque P, et al. In vivo visualization and ex vivo quantification of murine breast cancer cells in the mouse brain using MRI cell tracking and electron paramagnetic resonance. NMR in Biomedicine. 2015;28(3):367-375.

[19] Estelrich J, Sánchez-Martín J, Busquets A. Nanoparticles in magnetic resonance imaging: from simple to dual contrast agents. International Journal of Nanomedicine. 2015;10:1727-1741.

[20] Seyfer P, Pagenstcher A, Mandic R, et al. Cancer and inflammation: Differentiation by USPIO-enhanced MR imaging. Journal of Magnetic Resonance Imaging. 2014;39(3):665-672.

[21] Neuwelt A, Sidhu N, Hu C, et al. Iron-based superparamagnetic nanoparticle contrast agents for MRI of infection and inflammation. American Journal of Roentgenology. 2015;204(3):W302-W313.

[22] Kandasamy G, Maity D. Recent advances in superparamagnetic iron oxide nanoparticles (SPIONs) for in vitro and in vivo cancer nanotheranostics. International Journal of Pharmaceutics. 2015;496(2):191-218.

[23] Vidavsky N, Kunitake A, Chiou E, et al. Studying biomineralization pathways in a 3D culture model of breast cancer microcalcifications. Biomaterials. 2018;179:71-84.

[24] Talari S, Raza A, Rehman S, Rehman IU. Analyzing normal proliferating, hypoxic and necrotic regions of T-47D human breast cancer spheroids using Raman spectroscopy. Applied Spectroscopy Reviews. 2017;52(10):909-924.

[25] Khaniabadi M, Majik AMSA; Asif M, et al. Breast cancer cell targeted MR molecular imaging probe: Anti-MUC1 antibody-based magnetic nanoparticles. Journal of Physics: Conference Series. 2017;851:012014.

[26] Khaniabadi M, Shahbazi-Gahrouei D, Suhaimi M, et al. In vitro study of SPIONs-C595 as molecular imaging probe for specific breast cancer (MCF-7) cells detection. Iranian Biomedical Journal. 2017;21(6):360-368.

[27] Khaniabadi M, Shahbazi-Gahrouei D, Jafaar S, et al. Magnetic iron oxide nanoparticles as T2 MR imaging contrast agent for detection of breast cancer (MCF-7) cell. Avicenna Journal of Medical Biotechnology. 2017;9(4):181-188.

[28] Jafari F, Khadeer B, Iqbal A, et al. Increased aqueous solubility and proapoptotic activity of potassium koetjapate against human colorectal cancer cells. Journal of Pharmacy and Pharmacology. 2014;66(10):1394-1409.

[29] Funovics A, Kapeller B, Hoeller C, et al. MR imaging of the her2/neu and 9.2. 27 tumor antigens using immunospecific contrast agents. Magnetic Resonance Imaging. 2004;22(6):843-850.

[30] Oghabian M, Jeddi-Tehrani M, Zolfaghari A, et al. Detectability of Her2 positive tumors using monoclonal antibody conjugated iron oxide nanoparticles in MRI. Journal of Nanoscience and Nanotechnology. 2011;11(6):5340-5344.

[31] Arancibia S, Barrientos A, Torrejón J, et al. Copper oxide nanoparticles recruit macrophages and modulate nitric oxide, proinflammatory cytokines and PGE2 production through arginase activation. Nanomedicine. 2016;11(10):1237-1251.

[32] Zhang J, Ring L, Hurley R, et al. Quantification and biodistribution of iron oxide nanoparticles in the primary clearance organs of mice using T1 contrast for heating. Magnetic Resonance in Medicine. 2017;78(2):702-712.

[33] Rodríguez E, Simoes V, Roig A, et al. An iron-based T 1 contrast agent made of iron-phosphate complexes: In vitro and in vivo studies. Magnetic Resonance Materials in Physics, Biology and Medicine. 2007;20(1):27-37.

[34] Callaghan F, Mohammadi S, Weiskopf N. Synthetic quantitative MRI through relaxometry modelling. NMR in Biomedicine. 2016;29(12):1729-1738. 